Appl. Phys. B 50, 393-404 (1990)

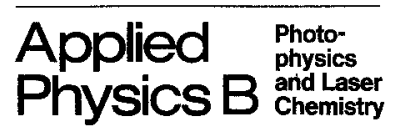

(C) Springer-Verlag 1990

\title{
Second Harmonic Generation in Planar Optical Waveguides
}

\author{
A. Bratz ${ }^{1}$, B. U. Felderhof ${ }^{1}$, and G. Marowsky ${ }^{2}$ \\ ${ }^{1}$ Institut für Theoretische Physik A, R.W.T.H. Aachen, Templergraben 55, \\ D-5100 Aachen, Fed. Rep. Germany \\ ${ }^{2}$ Max-Planck-Institut für biophysikalische Chemie, Postfach 2841, \\ D-3400 Göttingen 1, Fed. Rep. Germany
}

Received 14 July 1989/Accepted 17 November 1989

\begin{abstract}
We study optical second harmonic generation (SHG) in planar waveguide structures composed of several layers with different dielectric constants. We develop a general formalism for the calculation of mode generation by a planar antenna embedded in the waveguide. As an application we consider a monolayer of high second-order susceptibility adsorbed at the interface between two layers of the waveguide structure. Periodic modulation of the nonlinear susceptibility allows phase matching leading to dramatically enhanced second harmonic intensities. We investigate the SHG-efficiency of various experimentally realizable geometries.
\end{abstract}

PACS: $42.65 k, 42.80,42.82$

In recent years several devices have been proposed for optical second harmonic generation in planar waveguide structures. Extensive reviews of the various proposals have been presented by Stegeman and Seaton [1]. In this article we consider in particular second harmonic generation from a monolayer of optically nonlinear molecules adsorbed at an interface in the planar waveguide structure.

In one of the earliest proposals a nonlinear monolayer was deposited on the surface of a thin film waveguide [2]. In this article we consider more general planar waveguide structures with a view of optimizing the geometry for efficient second harmonic generation. We calculate the efficiency of conversion of an incident fundamental guided mode into a second harmonic guided mode propagating in the same direction. An optimized device, if realized, might be more effective than presently used inorganic frequency doublers such as crystals of KDP (potassium dihydrogen-phosphate) in which phase-matched bulk second harmonic generation occurs.

In the first few sections of this article we develop a formalism for the calculation of mode generation by a planar antenna embedded in a general waveguide structure. An alternative coupled wave formalism has been discussed by Sipe and Stegeman [3] in relation to a calculation of the total field. In our analysis the total field is represented by a Fourier integral and the amplitude of each of the generated normal modes is then found from a pole contribution to the integral. We present a detailed expression for the conversion efficiency in general planar waveguide structures.

We apply the theory to a calculation of SHG from a nonlinear monolayer adsorbed at an interface. The efficiency is enhanced by arranging the adsorbed molecules into a grating. This allows constructive interference of the second harmonic waves, provided phase matching can be achieved. We investigate how to optimize the enhancement by a proper choice of an experimentally realizable waveguide structure and of the location and dimensions of the adsorbate grating.

Up till now, waveguide SHG has usually been based on a phase-matching condition between guided modes at frequencies $\omega$ and $2 \omega$. The relevant theory was developed by Conwell [4], and an experimental realization has been demonstrated by Jain and Hewig [5]. The effect is discussed in a recent review by Williams [6]. Phase-matched SHG in a liquid-filled waveguide was realized experimentally by Levine et al. [7], who used a periodic electrode to modulate the 
nonlinear susceptibility. Phase-matched SHG in solid thin films with etched gratings was achieved by Chen et al. [8]. Phase-matching achieved by a grating structure obtained by periodic index-modulation of a planar waveguide has been proposed by Haus and Reider [9] . They have studied the enhancement to be expected from such a structure. Second harmonic generation from surface gratings has recently been studied by Reider et al. [10].

SHG in a channel waveguide resonator has been investigated by Regener and Sohler [11]. "Cerenkov"SHG was studied by Taniuchi and Yamamoto [12]. Grating-assisted SHG was studied by Jaskorzynska et al. [13]. Quasi-phase-matched SHG was studied by Lim et al. [14] and by Webjörn et al. [15].

\section{Planar Waveguide Theory}

We consider a stratified medium with frequencydependent dielectric profile $\varepsilon(z, \omega)$ and magnetic permeability profile $\mu(z, \omega)$. We assume that for the frequencies $\omega$ of interest $\varepsilon$ and $\mu$ are real. Also we assume that $\varepsilon$ and $\mu$ tend to constants $\varepsilon_{1}, \mu_{1}$ for $z \rightarrow-\infty$ and to constants $\varepsilon_{\mathrm{f}}, \mu_{\mathrm{f}}$ for $z \rightarrow+\infty$. An example of the geometry with our choice of coordinates is shown in Fig. 1. We consider plane wave solutions of Maxwell's equations which do not depend on the $y$-coordinate and which depend on $x$ and $t$ through a factor $\exp (i p x-i \omega t)$. Maxwell's equations for the electric and magnetic field amplitudes $\mathbf{E}(z)$ and $\mathbf{H}(z)$ then read in Gaussian units

$\mathrm{i} p \varepsilon E_{x}+\frac{d}{d z}\left(\varepsilon E_{z}\right)=0, \quad \mathrm{i} p \mu H_{x}+\frac{d}{d z}\left(\mu H_{z}\right)=0$,

$\frac{d E_{y}}{d z}=-\mathrm{i} k \mu H_{x}, \quad \frac{d H_{y}}{d z}=\mathrm{i} k \varepsilon E_{x}$,

$\frac{d E_{x}}{d z}-\mathrm{i} p E_{z}=\mathrm{i} k \mu H_{y}, \quad \frac{d H_{x}}{d z}-\mathrm{i} p H_{z}=-\mathrm{i} k \varepsilon E_{y}$,

$p E_{y}=k \mu H_{z} \quad p H_{y}=-k \varepsilon E_{z}$,

where $k=\omega / c$ is the vacuum wavenumber. The solutions of these equations may be decomposed according to two polarizations. For TE-polarization (s) the components $E_{x}, E_{z}$, and $H_{y}$ vanish and the equations may be combined into the single equation [16]

$\frac{d^{2} E_{y}}{d z^{2}}-\frac{1}{\mu} \frac{d \mu}{d z} \frac{d E_{y}}{d z}+\varepsilon \mu k^{2} E_{y}=p^{2} E_{y} \quad$ (TE)

For TM-polarization (p) the components $E_{y}, H_{x}$, and $H_{z}$ vanish and the equations may be combined into the single equation [16]

$\frac{d^{2} H_{y}}{d z^{2}}-\frac{1}{\varepsilon} \frac{d \varepsilon}{d z} \frac{d H_{y}}{d z}+\varepsilon \mu k^{2} H_{y}=p^{2} H_{y} \quad$ (TM) .

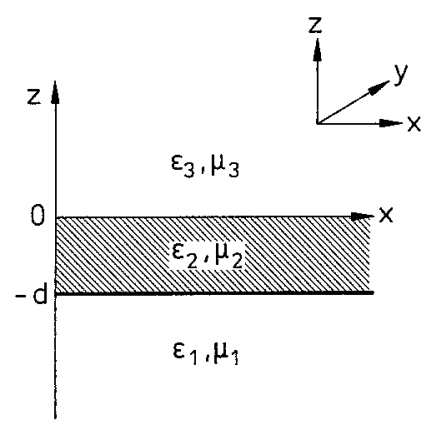

Fig. 1. Example of a stratified medium with our choice of coordinates. This situation is denoted as geometry I

We first consider TE-polarization. We assume that for $z<z_{1}$ the permeabilities $\varepsilon, \mu$ equal $\varepsilon_{1}, \mu_{1}$ and that for $z>z_{\mathrm{f}}$ they equal $\varepsilon_{\mathrm{f}}, \mu_{\mathrm{f}}$. We write the solution of (1.2) in these two asymptotic regions

$E_{y}(z)=a_{1}^{+} \mathrm{e}^{\mathrm{i} q_{1} z}+a_{1}^{-} \mathrm{e}^{-\mathrm{i} q_{1} z}$ for $z<z_{1}$,

$E_{\mathrm{y}}(z)=a_{\mathrm{f}}^{+} \mathrm{e}^{\mathrm{i} q_{\mathrm{f}} z}+a_{\mathrm{f}}^{-} \mathrm{e}^{-\mathrm{i} q_{\mathrm{f}} z}$ for $z>z_{\mathrm{f}}$,

where the wavenumbers $q_{1}$ and $q_{\mathrm{f}}$ are given by

$q_{j}=\sqrt{\varepsilon_{j} \mu_{j} k^{2}-p^{2}}$.

Clearly these are real only up to a maximum value of $p$ given by $\sqrt{\varepsilon_{j} \mu_{j}} k$ in either case, and are pure imaginary beyond this value. Through (1.2) the amplitudes in (1.4) are related by a transfer matrix $\underset{\approx}{W}$ according to [16]

$\left(\begin{array}{c}a_{\mathrm{f}}^{+} \\ a_{\mathrm{f}}^{-}\end{array}\right)=\left(\begin{array}{ll}W_{11} & W_{12} \\ W_{21} & W_{22}\end{array}\right)\left(\begin{array}{c}a_{1}^{+} \\ a_{1}^{-}\end{array}\right)$.

The guided mode solutions [17-19] occur at discrete values $\left\{p_{j}\right\}$ of $p$ larger than $\sqrt{\varepsilon_{\mathrm{m}} \mu_{\mathrm{m}}} k$, where $\varepsilon_{\mathrm{m}} \mu_{\mathrm{m}}$ is the maximum of $\varepsilon_{1} \mu_{1}$ and $\varepsilon_{\mathrm{f}} \mu_{\mathrm{f}}$. At these discrete values there are solutions of (1.2) which decay exponentially for $z<z_{1}$ and for $z>z_{\mathrm{f}}$. Hence $a_{\mathrm{f}}^{-}$and $a_{1}^{+}$ must vanish, and from (1.6) we find the relations

$a_{\mathrm{f}}^{+}=W_{12} a_{1}^{-}, \quad 0=W_{22} a_{1}^{-}$.

Thus for a nontrivial solution, the relation

$W_{22}(p, k)=0$

must be satisfied. At fixed $k$ the roots of this equation determine the discrete values $\left\{p_{j}\right\}$ for which a guided mode solution exists. We call $W_{22}(p, k)$ the dispersion function and refer to (1.8) as the waveguide condition.

We note that in the continuum $0<p<\sqrt{\varepsilon_{\mathrm{m}} \mu_{\mathrm{m}}} k$ the transfer matrix is related to the transmission and reflection coefficients of the planar structure by

$\underset{\approx}{W}=\frac{1}{T^{\prime}}\left(\begin{array}{cc}T T^{\prime}-R R^{\prime} & R^{\prime} \\ -R & 1\end{array}\right)$. 
The properties

$$
\left(\begin{array}{l}
T \\
0
\end{array}\right)=\underset{\approx}{W}\left(\begin{array}{l}
1 \\
R
\end{array}\right), \quad\left(\begin{array}{c}
R^{\prime} \\
1
\end{array}\right)=\underset{\approx}{W}\left(\begin{array}{c}
0 \\
T^{\prime}
\end{array}\right)
$$

provide the transfer relations between transmission and reflection coefficients for a wave incident from $z<z_{1}$ or from $z>z_{\mathrm{f}}$. The relation (1.8) therefore implies that the guided mode solutions occur at poles of the analytically continued transmission coefficient $T^{\prime}$. This is analogous to the condition for bound states in quantum mechanics [20].

The analogy with quantum mechanics may be made more evident by use of the transformation

$E_{y}(z)=\sqrt{\mu(z)} f(z)$.

By substitution we find that (1.2) is transformed to

$\frac{d^{2} f}{d z^{2}}-V(z) f=p^{2} f$

where the function $V(z)$ is given by

$V(z)=-\varepsilon \mu k^{2}+\sqrt{\mu} \frac{d^{2}}{d z^{2}} \frac{1}{\sqrt{\mu}}$.

By comparison with the Schrödinger equation we see that

$U(z)=\varepsilon_{\mathrm{m}} \mu_{\mathrm{m}} k^{2}+V(z)$

may be identified as the potential. The eigenvalues $\varepsilon_{\mathrm{m}} \mu_{\mathrm{m}} k^{2}-p_{j}^{2}$ then correspond to bound state energies.

It follows from (1.3) that for TM-polarization exactly the same relations hold if we replace $E_{y}$ by $H_{y}$ and $\varepsilon$ by $\mu$. Where necessary the symbols corresponding to the two types of solution will be distinguished by a superscript $\mathrm{E}$ or $\mathrm{M}$.

\section{Mathematical Properties}

In this section we deduce some mathematical properties which follow from the above equations. Again we consider only TE-polarization, the transcription to TM-polarization being obvious.

The form (1.12) of the wave equation has mathematical advantages. We define two fundamental solutions $f_{1}(z)$ and $f_{2}(z)$ by the requirements

$$
\begin{aligned}
& f_{1}(z)=\frac{1}{\sqrt{\mu_{1}}} \mathrm{e}^{\mathrm{i} q_{1} z} \\
& f_{2}(z)=\frac{1}{\sqrt{\mu_{1}}} \mathrm{e}^{-\mathrm{i} q_{1} z}
\end{aligned}
$$

By comparison with (1.6) we find that the asymptotic behavior of the solutions for $z>z_{\mathrm{f}}$ is given by

$$
\begin{aligned}
& f_{1}(z)=\frac{1}{\sqrt{\mu_{\mathrm{f}}}}\left[W_{11} \mathrm{e}^{\mathrm{i} q_{\mathrm{f}} z}+W_{21} \mathrm{e}^{-\mathrm{i} q_{\mathrm{f}} z}\right] \\
& f_{2}(z)=\frac{1}{\sqrt{\mu_{\mathrm{f}}}}\left[W_{12} \mathrm{e}^{\mathrm{i} q_{\mathrm{f}} z}+W_{22} \mathrm{e}^{-\mathrm{i} q_{\mathrm{f}} z}\right]
\end{aligned}
$$

From the form of (1.12) it follows that the Wronskian of the two solutions

$\Delta\left(f_{1}, f_{2}\right)=\left|\begin{array}{ll}f_{1} & f_{2} \\ f_{1}^{\prime} & f_{2}^{\prime}\end{array}\right|$

does not depend on $z$. Hence we find the relation

$W_{11} W_{22}-W_{12} W_{21}=\frac{\mu_{\mathrm{f}}}{\mu_{1}} \frac{q_{1}}{q_{\mathrm{f}}}$

Using (1.9) this yields the reciprocity relation

$\mu_{1} q_{\mathrm{f}} T=\mu_{\mathrm{r}} q_{1} T^{\prime}$

From the fact that the coefficients in (1.12) are real it follows that for $p^{2}$ in the interval $0 \leqq p^{2} \leqq \varepsilon_{1} \mu_{1} k^{2}$, where $q_{1}$ is real, the solutions $f_{1}$ and $f_{2}$ are each other's complex conjugate. Hence it follows from (2.2) that for values of $p$ for which $q_{\mathrm{f}}$ is also real the following relations hold

$W_{11}=W_{22}^{*}, \quad W_{12}=W_{21}^{*}$.

These relations are special cases of more general relations valid in the whole complex $p$-plane, namely

$W_{11}=\bar{W}_{22}, \quad W_{12}=\bar{W}_{21}$,

where the overhead bar indicates a "time-reversal" operation. The meaning of this operation will be made clear in Sect. 6.

It will be convenient to introduce a third fundamental solution by

$f_{3}(z)=\frac{1}{\sqrt{\mu_{\mathrm{f}}}} \mathrm{e}^{\mathrm{i} q_{\mathrm{f}} z}$ for $z>z_{\mathrm{f}}$.

It follows from (2.2) that, for the values $\left\{p_{j}\right\}$ for which the waveguide condition is satisfied, the solutions $f_{2}$ and $f_{3}$ are related by $f_{2}(z)=W_{12} f_{3}(z)$.

For later purposes it is of interest to derive an expression for the norm of the eigensolutions. Differentiating (1.12) with respect to $p^{2}$ one easily finds the identity

$\frac{\partial}{\partial z}\left[f \frac{\partial}{\partial z}\left(\frac{\partial f}{\partial p^{2}}\right)-\frac{\partial f}{\partial p^{2}} \frac{\partial f}{\partial z}\right]=f^{2}$.

Applying this identity to the eigensolution $f_{2 j}(z)$, making use of the waveguide condition (1.8), and 
integrating over $z$, one finds

$N_{j}=\int_{-\infty}^{\infty} f_{2 j}^{2} d z=\left.\frac{-\mathrm{i} q_{\mathrm{f} j}}{\mu_{\mathrm{f}} p_{j}} W_{12}\left(p_{j}\right) \frac{\partial W_{22}(p)}{\partial p}\right|_{p_{j}}$.

We shall show in the next section that the norm $N_{j}$ is related to the intensity of the mode. We note that for real $p$-values for which $q_{1}$ and $q_{\mathrm{f}}$ are pure imaginary the functions $W_{12}(p)$ and $W_{22}(p)$ are real.

\section{Excitation of Guided Modes}

In this section we return to the physical situation and describe how the eigenmodes may be excited by an oscillating dipole density. We begin by relating the norm of an eigenmode, given by the expression (2.10), to the physical intensity. The energy current density averaged over a time period $2 \pi / \omega$ is given by the Poynting vector

$\mathbf{S}=\frac{c}{8 \pi} \operatorname{Re}\left(\mathbf{E} \times \mathbf{H}^{*}\right)$.

The $x$-component of this expression may be decomposed into

$S_{x}=S_{x}^{\mathrm{E}}+S_{x}^{\mathrm{M}}$,

with the separate terms for TE- and TM-polarization

$S_{x}^{\mathrm{E}}=\frac{c}{8 \pi} \operatorname{Re}\left\{E_{y} H_{z}^{*}\right\}, \quad S_{x}^{\mathrm{M}}=\frac{-c}{8 \pi} \operatorname{Re}\left\{E_{z} H_{y}^{*}\right\}$.

We define the total intensity by the integrals

$J^{\mathrm{E}}(x)=\int_{-\infty}^{\infty} S_{x}^{\mathrm{E}}(x, z) d z, \quad J^{\mathrm{M}}(x)=\int_{-\infty}^{\infty} S_{x}^{\mathrm{M}}(x, z) d z$.

For a single eigenmode of either TE- or TM-type the expressions (3.3) become

$S_{x j}^{\mathrm{E}}=\frac{c p_{j}^{\mathrm{E}}}{8 \pi k} \frac{\left|E_{y j}\right|^{2}}{\mu}, \quad S_{x j}^{\mathrm{M}}=\frac{c p_{j}^{\mathrm{M}}}{8 \pi k} \frac{\left|H_{y j}\right|^{2}}{\varepsilon}$

which are independent of $x$. By comparison with (1.11) and (2.10) we find that for a single eigenmode excited with amplitude $a_{1 j}^{-}$the intensity is related to the norm of the mode by

$J_{j}=\frac{c p_{j}}{8 \pi k} N_{j}\left|a_{1 j}^{-}\right|^{2}$.

This expression is formally the same for both polarizations.

Using orthogonality of the eigenmodes [17-19] we find that for a linear superposition of guided modes, all oscillating at the same frequency $\omega$, the total intensity is given by

$J=\sum_{j}\left(J_{j}^{\mathrm{E}}+J_{j}^{\mathrm{M}}\right)$

which again does not depend on $x$.
Next we investigate how radiation is emitted by an antenna in close contact with or embedded in the waveguide. We consider a surface polarization $[21,22]$ $\mathbf{P}^{\mathbf{S}}(x)$ of finite extent in the $x$-direction, independent of $y$, located in the plane $z=z_{0}$, and oscillating at frequency $\omega$. The corresponding charge and current densities are

$\varrho(\mathbf{r})=-\frac{\partial P_{x}^{\mathrm{S}}}{\partial x} \delta\left(z-z_{0}\right)-P_{z}^{\mathrm{S}} \delta^{\prime}\left(z-z_{0}\right)$,

$\mathbf{j}(\mathbf{r})=-\mathrm{i} \omega \mathbf{P}^{\mathbf{S}}(x) \delta\left(z-z_{0}\right)$,

which must be added as source terms to Maxwell's equations. A Fourier analysis of the surface polarization yields

$\mathbf{P}^{\mathrm{S}}(x)=\int \hat{\mathbf{P}}^{\mathrm{S}}(p) \mathrm{e}^{\mathrm{i} p x} d p$.

From Maxwell's equations we now find instead of (1.2)

$$
\begin{aligned}
& \frac{d^{2} E_{y}}{d z^{2}}-\frac{1}{\mu} \frac{d \mu}{d z} \frac{d E_{y}}{d z}+\left(\varepsilon \mu k^{2}-p^{2}\right) E_{y} \\
& =-4 \pi k^{2} \mu \widehat{P}_{y}^{\mathrm{S}} \delta\left(z-z_{0}\right) .
\end{aligned}
$$

Similarly we find instead of (1.3)

$$
\begin{aligned}
& \frac{d^{2} H_{y}}{d z^{2}}-\frac{1}{\varepsilon} \frac{d \varepsilon}{d z} \frac{d H_{y}}{d z}+\left(\varepsilon \mu k^{2}-p^{2}\right) H_{y} \\
& =4 \pi \mathrm{i} k\left[\hat{P}_{x}^{\mathrm{s}} \delta^{\prime}\left(z-z_{0}\right)-\frac{1}{\varepsilon} \frac{d \varepsilon}{d z} \hat{P}_{x}^{\mathrm{S}} \delta\left(z-z_{0}\right)\right] \\
& \quad+4 \pi k p \hat{P}_{z}^{\mathrm{S}} \delta\left(z-z_{0}\right) .
\end{aligned}
$$

The solutions of these equations may be found with the aid of the Green's functions $G^{\mathrm{E}}\left(z, z_{0}\right)$ and $G^{\mathrm{M}}\left(z, z_{0}\right)$ defined by the equation

$\frac{d^{2} G}{d z^{2}}-V(z) G=p^{2} G+\delta\left(z-z_{0}\right)$.

The Green's function $G$ may be expressed in terms of the fundamental solutions defined in the preceding section. It is given by [23]

$G\left(z, z_{0}\right)=\frac{f_{2}\left(z_{<}\right) f_{3}\left(z_{>}\right)}{\Delta\left(f_{2}, f_{3}\right)}$,

where $z_{<}\left(z_{>}\right)$is the smaller (larger) of $z$ and $z_{0}$. The solution of (3.10) is given by

$$
E_{y}(z)=-4 \pi k^{2}[\mu(z)]^{1 / 2} G^{\mathrm{E}}\left(z, z_{0}\right)\left[\mu\left(z_{0}\right)\right]^{1 / 2} \hat{P}_{y}^{\mathrm{S}} .
$$

The solution of (3.11) is given by

$$
\begin{aligned}
H_{y}(z)= & -4 \pi \mathrm{i} k[\varepsilon(z)]^{1 / 2}\left[\frac{\partial G^{\mathrm{M}}\left(z, z_{0}\right)}{\partial z_{0}}\right. \\
& \left.+\frac{1}{2} \frac{d \ln \varepsilon\left(z_{0}\right)}{d z_{0}} G^{\mathrm{M}}\left(z, z_{0}\right)\right]\left[\varepsilon\left(z_{0}\right)\right]^{-1 / 2} \hat{P}_{x}^{\mathrm{S}} \\
& +4 \pi p k[\varepsilon(z)]^{1 / 2} G^{\mathrm{M}}\left(z, z_{0}\right)\left[\varepsilon\left(z_{0}\right)\right]^{-1 / 2} \hat{P}_{z}^{\mathrm{S}} .
\end{aligned}
$$


From (3.13) and (3.14) we find that $E_{y}(z)$ has the following discontinuities at $z_{0}$

$\left[E_{y}\right]=0, \quad\left[\frac{d E_{y}}{d z}\right]=-4 \pi k^{2} \mu \hat{P}_{y}^{\mathrm{s}}$.

Similarly $H_{y}(z)$ has the discontinuities

$\left[H_{y}\right]=4 \pi \mathrm{i} k \hat{P}_{x}^{\mathrm{s}}, \quad\left[\frac{d H_{y}}{d z}\right]=4 \pi k p \hat{P}_{z}^{\mathrm{s}}$.

These discontinuities are in agreement with the jump conditions [Ref. 21, Eq. (8)].

For a surface polarization $\mathbf{P}^{\mathrm{S}}(x)$ with arbitrary variation in the $x$-direction the fields $E_{y}(x, z)$ and $H_{y}(x, z)$ are now obtained by Fourier superposition. Thus we find

$E_{y}(x, z)=\int E_{y}(p, z) \mathrm{e}^{\mathrm{i} p x} d p$,

$H_{y}(x, z)=\int H_{y}(p, z) \mathrm{e}^{\mathrm{i} p x} d p$,

where $E_{y}(p, z)$ and $H_{y}(p, z)$ are given by (3.14) and (3.15) in terms of $\widehat{\mathbf{P}}^{\mathbf{S}}(p)$. It follows from (2.2) and (2.7) that the denominator in the Green's function (3.13) takes the form

$\Delta\left(f_{2}^{\mathrm{E}}, f_{3}^{\mathrm{E}}\right)=\frac{2 \mathrm{i} q_{\mathrm{f}}}{\mu_{\mathrm{f}}} W_{22}^{\mathrm{E}}(p, k)$,

$\Delta\left(f_{2}^{\mathrm{M}}, f_{3}^{\mathrm{M}}\right)=\frac{2 \mathrm{i} q_{\mathrm{f}}}{\varepsilon_{\mathrm{f}}} W_{22}^{\mathrm{M}}(p, k)$.

Hence the denominator vanishes at the eigenvalues $\left\{p_{j}^{\mathrm{E}}\right\}$ and $\left\{p_{j}^{\mathrm{M}}\right\}$, respectively. For large $x$ the contribution from the corresponding poles dominates the integrals in (3.18). This allows us to evaluate the amplitude of the various guided modes excited by the oscillating surface polarization.

\section{Emitted Radiation}

In this section we analyze the radiation emitted by a planar antenna, as introduced in the preceding section, in more detail. We are interested in the radiation channeled into the waveguide and detectable at large positive $x$. At sufficiently large distances from the antenna, i.e. after the decay of transients corresponding to evanescent wave solutions, the behavior of the fields is dominated by pole contributions to the integrals corresponding to roots of the waveguide condition (1.8). The contributions may be found by contour integration in (3.18) with the poles at positive $\left\{p_{j}\right\}$ shifted slightly upwards into the complex plane and those at $\left\{-p_{j}\right\}$ shifted slightly downwards. Thus we find for large positive $x$

$E_{y}(x, z) \approx \sum_{j} a_{1 j}^{-\mathrm{E}} \psi_{j}^{\mathrm{E}}(z) \exp \left(\mathrm{i} p_{j} x\right)$,

where we employ the notation

$\psi_{j}^{\mathrm{E}}(z)=\sqrt{\mu(z)} f_{2 j}^{\mathrm{E}}(z)$.
The amplitudes are given by

$a_{1 j}^{-\mathrm{E}}=4 \pi^{2} \mathrm{i} k^{2}\left(p_{j}^{\mathrm{E}} N_{j}^{\mathrm{E}}\right)^{-1} \psi_{j}^{\mathrm{E}}\left(z_{0}\right) \hat{P}_{y}^{\mathrm{S}}\left(p_{j}^{\mathrm{E}}\right)$,

where we have used (2.10). From (4.1) we may evaluate the intensity defined in (3.4). Because of the orthogonality of the different modes [17-19] there are no cross terms and we find

$J^{\mathrm{E}}=\sum_{j} J_{j}^{\mathrm{E}}=2 \pi^{3} c k^{3} \sum_{j}\left(p_{j}^{\mathrm{E}} N_{j}^{\mathrm{E}}\right)^{-1}\left[\psi_{j}^{\mathrm{E}}\left(z_{0}\right)\right]^{2}\left|\hat{P}_{y}^{\mathrm{S}}\left(p_{j}^{\mathrm{E}}\right)\right|^{2}$.

Similarly we find for large positive $x$

$H_{y}(x, z) \approx \sum_{j} a_{1 j}^{-\mathrm{M}} \psi_{j}^{\mathrm{M}}(z) \exp \left(\mathrm{i} p_{j} x\right)$

with the notation

$\psi_{j}^{\mathrm{M}}(z)=\sqrt{\varepsilon(z)} f_{2 j}^{\mathrm{M}}(z)$.

The amplitudes are given by

$\begin{aligned} a_{1 j}^{-\mathrm{M}}= & -4 \pi^{2} k\left(p_{j}^{\mathrm{M}} N_{j}^{\mathrm{M}}\right)^{-1} \frac{1}{\varepsilon\left(z_{0}\right)} \frac{d \psi_{j}^{\mathrm{M}}\left(z_{0}\right)}{d z_{0}} \hat{P}_{x}^{\mathrm{S}}\left(p_{j}^{\mathrm{M}}\right) \\ & -4 \pi^{2} i k\left(N_{j}^{\mathrm{M}}\right)^{-1} \frac{\psi_{j}^{\mathrm{M}}\left(z_{0}\right)}{\varepsilon\left(z_{0}\right)} \hat{P}_{z}^{\mathrm{S}}\left(p_{j}^{\mathrm{M}}\right) .\end{aligned}$

This yields for the intensity

$$
\begin{aligned}
J^{\mathrm{M}}= & \sum_{j} J_{j}^{\mathrm{M}} \\
= & 2 \pi^{3} c k \sum_{j}\left(p_{j}^{\mathrm{M}} N_{j}^{\mathrm{M}}\right)^{-1} \mid \frac{1}{\varepsilon\left(z_{0}\right)} \frac{d \psi_{j}^{\mathrm{M}}\left(z_{0}\right)}{d z_{0}} \hat{P}_{x}^{\mathrm{S}}\left(p_{j}^{\mathrm{M}}\right) \\
& +\left.\mathrm{i} p_{j}^{\mathrm{M}} \frac{\psi_{j}^{\mathrm{M}}\left(z_{0}\right)}{\varepsilon\left(z_{0}\right)} \hat{P}_{z}^{\mathrm{S}}\left(p_{j}^{\mathrm{M}}\right)\right|^{2} .
\end{aligned}
$$

The expressions (4.4) and (4.8) have a fairly simple structure. The efficiency with which a surface polarization $\mathbf{P}^{\mathbf{S}}(x)$ excites the guided modes is determined by its Fourier component at the mode wavenumber $p_{j}$, as well as by its location $z_{0}$ via the eigenfunction $\psi_{j}\left(z_{0}\right)$, which appears quadratically with its proper normalization $N_{j}$.

\section{Phase-Matched Second Harmonic Generation}

In this section we discuss the principle of secondharmonic generation by use of a phase-matched adsorbate in close contact with or embedded in a planar waveguide. In practice the adsorbate consists of optically nonlinear molecules located at an interface. We assume the general type of geometry discussed in the preceding section and consider a surface polarization $\mathbf{P}^{\mathbf{S}}(x)$ which is induced by an incident fundamental wave and which acts as an antenna emitting waves at the second harmonic frequency [24]. Thus we put

$\mathbf{P}^{\mathrm{S}}(x)=\chi^{(3)}(x): \mathbf{E}_{10}(x) \mathbf{E}_{10}(x)$, 


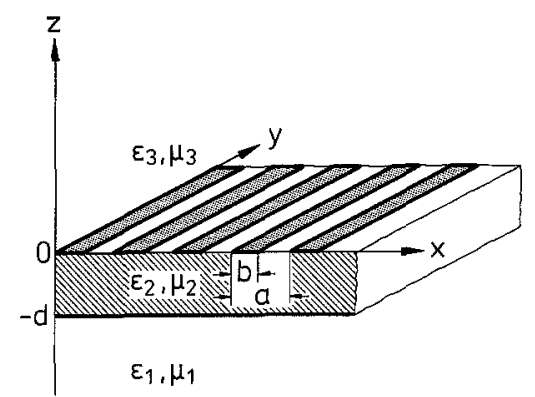

Fig. 2. Example of the grating and waveguide structure

where $\mathbf{E}_{10}(x)$ is the incident fundamental field. Of course, if the fundamental field oscillates with frequency $\omega$, then the surface polarization oscillates at frequency $2 \omega$ and this must be taken into account in the expressions of the preceding sections. We assume that the adsorbate is so weak that it does not disturb the fundamental wave. This is expressed by the subscript zero in (5.1).

The fundamental wave is a linear combination of guided modes with $x$-dependence $\exp \left(\mathrm{i} p_{j} x\right)$ with wavenumber $p_{j}(\omega)$. We shall assume that the susceptibility tensor $\chi^{(3)}$ depends on $x$ via the density of adsorbed molecules. If the adsorbate has a periodicity $a$ characterized by the wavenumber $K=2 \pi / a$, then we may expect resonance when the phase-matching condition

$p_{j}(2 \omega)=2 p_{k}(\omega)+n K, \quad n=0, \pm 1, \pm 2, \ldots$

is satisfied. More specifically it is natural to aim at satisfying the condition

$p_{0}(2 \omega)=2 p_{0}(\omega) \pm K$,

for the lowest mode $j=0$. We shall call this the Bragg condition.

We consider in particular a grating of period $a$ consisting of $N$ adsorbate stripes of width $b<a$. An example of the grating and waveguide is shown in Fig. 2. The susceptibility function is given by

$\chi(x)=\chi g_{N}(x)$,

with the geometric function

$g_{N}(x)=\sum_{n=0}^{N-1} \theta\left(b, x-n a-\frac{1}{2} b\right)$,

where

$$
\begin{aligned}
\theta(b, x) & =1 \text { for }-\frac{b}{2}<x<\frac{b}{2} \\
& =0 \text { for }|x|>\frac{b}{2} .
\end{aligned}
$$

The prefactor $\chi$ in (5.4) is a third rank tensor independent of $x$. We shall assume that $\chi$ has the characteristics of a layer isotropic about the surface normal (in the $z$-direction). We also assume that there exists a mirror plane containing the $z$-axis to exclude chirality. From these assumptions it follows that $\chi$ has only three independent components (for details see [25-27]). The Fourier-component $\mathbf{P}^{\mathbf{S}}(p)$ is proportional to

$$
\begin{aligned}
\hat{G}_{N}\left(p-2 p_{k}(\omega)\right) & =\frac{1}{2 \pi} \int_{-\infty}^{\infty} g_{N}(x) \mathrm{e}^{2 \mathrm{i} p_{k}(\omega) x-\mathrm{i} p x} d x \\
& =\frac{\exp \left(\mathrm{i} s_{k} b\right)-1}{2 \pi \mathrm{i} s_{k}} \frac{1-\exp \left(\mathrm{i} N s_{k} a\right)}{1-\exp \left(\mathrm{i} s_{k} a\right)},
\end{aligned}
$$

where we have introduced the variable

$s_{k}=2 p_{k}(\omega)-p$.

For real $p$ the absolute square of the second factor in (5.7) is given by

$F_{N}(s a)=\left|\frac{1-\mathrm{e}^{\mathrm{i} N s a}}{1-\mathrm{e}^{\mathrm{i} s a}}\right|^{2}=\frac{\sin ^{2}(N s a / 2)}{\sin ^{2}(s a / 2)}$

which takes the value $N^{2}$ at $s a=2 n \pi$, where $n=0, \pm 1, \pm 2, \ldots$. Since we wish $\widehat{p}^{\mathrm{S}}(p)$ to be maximum at $p_{0}(2 \omega)$ we choose the lattice distance $a$ such that

$a=2 \pi\left|2 p_{0}(\omega)-p_{0}(2 \omega)\right|^{-1}$

corresponding to $n=1$ or $n=-1$. In this way we satisfy the Bragg condition (5.3). The width of the function in (5.9) at $s= \pm 2 \pi / a$ is of order $1 / N a$. Hence the area of the peak is proportional to $N$. The larger $N$ the more precisely the condition (5.10) must be satisfied. An error $\Delta a$ in the value of $a$ implies an error $\Delta p=2 \pi \Delta a / a^{2}$ in $p$-space. If we require this to be at most $1 / N a$, then $N$ cannot be larger than $a / 2 \pi \Delta a$. Ideally one would use a tunable laser and adjust the frequency $\omega$ such that the condition (5.10) is precisely satisfied. With that choice the absolute square of the first factor in (5.7) is maximal at $b=\frac{1}{2} a$.

\section{Specific Geometries}

In this section we describe three geometrical structures which we shall investigate with regard to their efficiency for second harmonic generation. The simplest geometry under consideration is depicted in Fig. 1. Here the waveguide structure consists of a layer of thickness $d$ with dielectric constant $\varepsilon_{2}$ sandwiched between two halfspaces with dielectric constants $\varepsilon_{1}$ and $\varepsilon_{3}$. In practice we consider one of the halfspaces to be air and in that case we put $\varepsilon_{3}=1$. The grating of adsorbed molecules is located at the interface between media 2 and 3. We choose the dielectric constants such that $\varepsilon_{2}>\varepsilon_{1} \geqq \varepsilon_{3}$. In that case the layer $\varepsilon_{2}$ may act as a plane waveguide. We denote the structure shown in Fig. 1 as geometry $I$. 


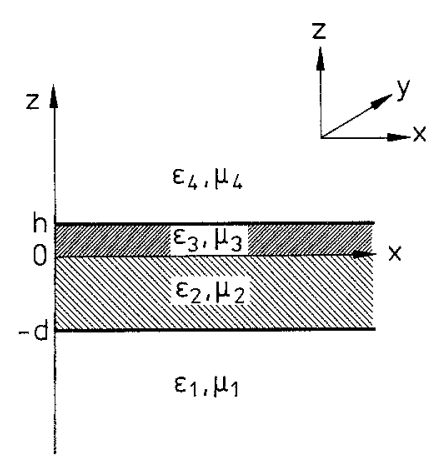

Fig. 3. Geometry II. In the explicit calculations we take the limit $h \rightarrow 0+$ and put $\varepsilon_{4}=\varepsilon_{1}$

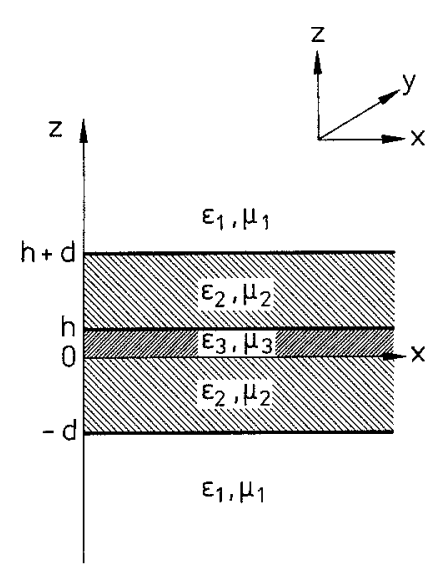

Fig. 4. Geometry III. In the explicit calculations we take the limit $h \rightarrow 0+$

The second structure we consider is depicted in Fig. 3. In this case the structure of geometry $I$ is modified such that medium 3 has a finite thickness $h$, beyond which there is a halfspace with dielectric constant $\varepsilon_{4}$. In our explicit calculations we put $\varepsilon_{4}=\varepsilon_{1}$. We denote this structure as geometry II. If $h$ is comparable with or smaller than $d$, then the field amplitude of the fundamental at the adsorbed molecules can be much larger than in geometry $I$. This leads to enhanced second harmonic generation.

Finally we consider the symmetric structure shown in Fig. 4. This case is a modification of geometry II. We take $h$ to be small, i.e. much less than $d$ and the wavelength of the fundamental. Medium 4 is replaced by a layer of thickness $d$ with dielectric constant $\varepsilon_{2}$ and a halfspace with dielectric constant $\varepsilon_{1}$. We denote this structure as geometry III. We shall show that with respect to SHG efficiency geometry III is the most favourable one from a theoretical point of view.

In all three geometries the wave equations (1.2) and (1.3) may be solved by the transfer matrix method [16, 21]. We briefly recall the basic equations in the notation of this article. In the layer $z_{j-1}<z<z_{j}$ the fields are written

$E_{y}(x, z)=\left[a_{j}^{+\mathrm{E}} \exp \left(\mathrm{i} q_{j} z\right)+a_{j}^{-\mathrm{E}} \exp \left(-\mathrm{i} q_{j} z\right)\right] \exp (\mathrm{ipx})$,

$H_{y}(x, z)=\left[a_{j}^{+\mathrm{M}} \exp \left(\mathrm{i} q_{j} z\right)+a_{j}^{-\mathrm{M}} \exp \left(-\mathrm{i} q_{j} z\right)\right] \exp (\mathrm{i} p x)$.

The amplitudes $a_{j}^{+}, a_{j}^{-}$below the discontinuity at $z_{j}$ may be related to the amplitudes $a_{j+1}^{+}, a_{j+1}^{-}$above by a $2 \times 2$ transfer matrix

$\left(\begin{array}{c}a_{j+1}^{+} \\ a_{j+1}^{-}\end{array}\right)=\underset{\approx}{R}\left(\begin{array}{l}a_{j}^{+} \\ a_{j}^{-}\end{array}\right)$.

The transfer matrix $\underset{\approx}{R_{j}}$ may be written as

$\underset{\approx}{R}={\underset{\approx}{U}}_{j}^{\prime} M_{j} U_{j}$

with the diagonal matrices

$U_{z j}^{\prime}=\left(\begin{array}{cc}\exp \left(-\mathrm{i} q_{j+1} z_{j}\right. & 0 \\ 0 & \exp \left(\mathrm{i} q_{j+1} z_{j}\right)\end{array}\right)$,
$U_{j}=\left(\begin{array}{cc}\exp \left(\mathrm{i} q_{j} z_{j}\right) & 0 \\ 0 & \exp \left(-\mathrm{i} q_{j} z_{j}\right)\end{array}\right)$

and with a matrix $M_{j}$ which is independent of position. The matrix $\underset{\approx}{M_{j}}$ is given by

$\underset{\approx j}{M_{j}}=\frac{1}{t_{j+1, j}}\left(\begin{array}{cc}1 & r_{j+1, j} \\ r_{j+1, j} & 1\end{array}\right)$

with Fresnel coefficients $t_{i j}$ and $r_{i j}$ which are

$t_{\mathrm{i} j}^{\mathrm{E}}=\frac{2 \mu_{j} q_{\mathrm{i}}}{\mu_{\mathrm{i}} q_{j}+\mu_{j} q_{\mathrm{i}}}, \quad r_{\mathrm{i} j}^{\mathrm{E}}=\frac{\mu_{j} q_{\mathrm{i}}-\mu_{\mathrm{i}} q_{j}}{\mu_{\mathrm{i}} q_{j}+\mu_{j} q_{\mathrm{i}}}$

for TE-polarization, and

$t_{\mathrm{i} j}^{\mathrm{M}}=\frac{2 \varepsilon_{j} q_{\mathrm{i}}}{\varepsilon_{\mathrm{i}} q_{j}+\varepsilon_{j} q_{\mathrm{i}}}, \quad r_{\mathrm{i} j}^{\mathrm{M}}=\frac{\varepsilon_{j} q_{\mathrm{i}}-\varepsilon_{\mathrm{i}} q_{j}}{\varepsilon_{\mathrm{i}} q_{j}+\varepsilon_{j} q_{\mathrm{i}}}$

for TM-polarization. The transmission of a wave through an array of $l$ discontinuities is given by the product

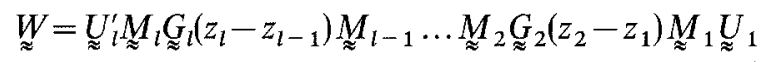

with the propagation matrix for medium $\varepsilon_{j}, \mu_{j}$

$G_{z j}(z)=\left(\begin{array}{cc}\exp \left(\mathrm{i} q_{j} z\right) & 0 \\ 0 & \exp \left(-\mathrm{i} q_{j} z\right)\end{array}\right)$.

The complete transfer matrix $W$ may be written in the form (1.9). The time-reversal operation mentioned below (2.7) is performed by replacing all wavenumbers $q$ by $-q$.

For each of the three geometries one may calculate the intensity $J_{j}^{\sigma}(2 \omega)$ of second harmonic generation in mode $j$ of polarization $\sigma$ for given intensity $J_{k}^{\sigma^{\prime}}(\omega)$ of the 
fundamental. The ratio of these two quantities defines the conversion coefficient

$\eta_{j k}^{\sigma \sigma^{\prime}}=J_{j}^{\sigma}(2 \omega) / J_{k}^{\sigma^{\prime}}(\omega)$.

In numerical calculations we consider only $\eta_{00}^{\sigma \sigma^{\prime}}$.

\section{Explicit Results}

In this section we present explicit results for the three geometries discussed above. For each of the three geometries we list the expressions for the functions $W_{12}(p, k), W_{22}(p, k)$ as well as the product $W_{12} W_{22}^{\prime}$ at $p_{j}$ from which the norm of the wavefunction may be evaluated according to (2.10). We recall the definitions (4.2) and (4.6) of the wavefunctions $\psi_{j}^{\mathrm{E}}(z)$ and $\psi_{j}^{\mathrm{M}}(z)$ in terms of $f_{2 j}^{\mathrm{E}}(z)$ and $f_{2 j}^{\mathrm{M}}(z)$. As before we omit the polarization index where possible. At the end of the section we also give the value of the wavefunction and its derivative at the position of the adsorbate.
Here the prime indicates differentiation with respect to $p$. The explicit expression for $\alpha_{j k}^{\mathrm{E}}$ is

$\alpha_{j k}^{\mathrm{E}}=k^{2} \mu_{j} \mu_{k} \frac{\varepsilon_{j} \mu_{j}-\varepsilon_{k} \mu_{k}}{\left(\mu_{k} q_{j}\right)^{2}-\left(\mu_{j} q_{k}\right)^{2}}$

and similarly for $\alpha_{j k^{\mathrm{M}}}^{\mathrm{M}}$. Of course, everywhere on the right in (7.5) one must put $p=p_{j}$. We do not indicate this explicitly.

Similarly we find for geometry II depicted in Fig. 3

$$
\begin{aligned}
W_{12}= & \frac{\mathrm{e}^{\mathrm{i}\left(q_{1}-q_{2}\right) d-\mathrm{i}\left(q_{3}+q_{4}\right) h}}{t_{43} t_{32} t_{21}}\left[\left(r_{32}+r_{21} \mathrm{e}^{2 \mathrm{i} q_{2} d}\right) \mathrm{e}^{2 \mathrm{i} q_{3} h}\right. \\
& \left.+r_{43}\left(1+r_{32} r_{21} \mathrm{e}^{2 \mathrm{i} q_{2} d}\right)\right], \\
W_{22}= & \frac{\mathrm{e}^{\mathrm{i}\left(q_{1}-q_{2}\right) d-\mathrm{i}\left(q_{3}-q_{4}\right) h}}{t_{43} t_{32} t_{21}}\left[1+r_{32} r_{21} \mathrm{e}^{2 \mathrm{i} q_{2} d}\right. \\
& \left.+r_{43}\left(r_{32}+r_{21} \mathrm{e}^{2 \mathrm{i} q_{2} d}\right) \mathrm{e}^{2 \mathrm{i} q_{3} h}\right] .
\end{aligned}
$$

For the product $W_{12} W_{22}^{\prime}$ at $p_{j}$ we find

$$
\begin{aligned}
\left.W_{12} \frac{\partial W_{22}}{\partial p}\right|_{p_{j}}= & \frac{-2 t_{34} \mathrm{e}^{-2 \kappa_{1} d}}{t_{43}\left|t_{32}\right|^{2}\left|t_{21}\right|^{2}} \sin \varphi_{32}\left[\varphi_{21}^{\prime}+2 q_{2}^{\prime} d\right. \\
& \left.+\frac{\left(1-r_{43}^{2} \mathrm{e}^{-4 \kappa_{3} h}\right) \varphi_{32}^{\prime}-2\left(r_{43}^{\prime}-2 \kappa_{3}^{\prime} h r_{43}\right) \mathrm{e}^{-2 \kappa_{3} h} \sin \varphi_{32}}{1+r_{43}^{2} \mathrm{e}^{-4 \kappa_{3} h}+2 r_{43} \mathrm{e}^{-2 \kappa_{3} h} \cos \varphi_{32}}\right] .
\end{aligned}
$$

We note that in all three geometries the wavenumbers $q_{j}$ for the guided modes are pure imaginary for $j=1,3,4$, whereas $q_{2}$ is real. Hence we may write

$q_{1}=\mathrm{i} \kappa_{1}, \quad q_{3}=\mathrm{i} \kappa_{3}, \quad q_{4}=\mathrm{i} \kappa_{4}$.

In addition we may put

$r_{21}=\mathrm{e}^{\mathrm{i} \varphi_{21}}, \quad r_{32}=\mathrm{e}^{\mathrm{i} \varphi_{32}}$

with real phase angles $\varphi_{21}$ and $\varphi_{32}$. This implies the identities

$\frac{r_{21}}{t_{21}^{2}}=\frac{1}{\left|t_{21}\right|^{2}}, \quad \frac{r_{32}}{t_{32}^{2}}=\frac{1}{\left|t_{32}\right|^{2}}$.

For geometry I, as depicted in Fig. 1, the elements $W_{12}$ and $W_{22}$ in this geometry are given by

$$
\begin{aligned}
& W_{12}=\frac{\mathrm{e}^{\mathrm{i}\left(q_{1}-q_{2}\right) d}}{t_{32} t_{21}}\left[r_{32}+r_{21} \mathrm{e}^{2 \mathrm{i} q_{2} d}\right], \\
& W_{22}=\frac{\mathrm{e}^{\mathrm{i}\left(q_{1}-q_{2}\right) d}}{t_{32} t_{21}}\left[1+r_{32} r_{21} \mathrm{e}^{2 \mathrm{i} q_{2} d}\right] .
\end{aligned}
$$

For the product $W_{12} W_{22}^{\prime}$ at $p_{j}$ we find

$$
\begin{aligned}
\left.W_{12} \frac{\partial W_{22}}{\partial p}\right|_{p_{j}}= & \frac{2 p_{j}}{q_{2}} \frac{\left|t_{23}\right|}{\left|t_{32}\right|\left|t_{21}\right|^{2}} \\
& \times \mathrm{e}^{-2 \kappa_{1} d}\left[d+\frac{\alpha_{12}}{\kappa_{1}}+\frac{\alpha_{32}}{\kappa_{3}}\right],
\end{aligned}
$$

where $\alpha_{j k}$ is defined by

$\alpha_{j k}=\frac{q_{j} q_{k}}{2 p} \frac{r_{j k}^{\prime}}{r_{j k}}$
Finally we consider geometry III depicted in Fig. 4. We simplify the expressions by taking the limit $h=0+$. This yields

$$
\begin{aligned}
& W_{12}=\frac{2 \mathrm{i}_{21}}{t_{12} t_{21}} \sin 2 q_{2} d, \\
& W_{22}=\frac{\mathrm{e}^{2 \mathrm{i}\left(q_{1}-q_{2}\right) d}}{t_{12} t_{21}}\left[1-r_{21}^{2} \mathrm{e}^{4 \mathrm{i} q_{2} d}\right] .
\end{aligned}
$$

For the product $W_{12} W_{22}^{\prime}$ at $p_{j}$ we find

$\left.W_{12} \frac{\partial W_{22}}{\partial p}\right|_{p_{j}}=\frac{4 p_{j}}{q_{2}} \frac{\mathrm{e}^{-2 \kappa_{1 d}}}{\left|t_{12}\right|\left|t_{21}\right|}\left[d+\frac{\alpha_{12}}{\kappa_{1}}\right]$.

In all three geometries we choose coordinates such that the interface between media 2 and 3 is at $z=0$. The adsorbate is located just above at $z=0+$. The wavefunction and its derivative at $z=0+$ are given by expressions which are the same for the three geometries. For the value of the wavefunction we find

$\psi(0+)=\mathrm{e}^{-\kappa_{1} d} \frac{\cos \left(\frac{1}{2} \varphi_{21}+q_{2} d\right)}{\cos \frac{1}{2} \varphi_{21}}$

and for its derivative

$\psi^{\prime}(0+)=-\kappa_{3} \frac{\left|t_{23}\right|}{\left|t_{32}\right|} \mathrm{e}^{-\kappa_{1} d} \frac{\sin \left(\frac{1}{2} \varphi_{21}+q_{2} d\right)}{\cos \frac{1}{2} \varphi_{21}}$.

The expressions presented in this section may be used for an explicit calculation of the intensity radiated by a polarization sheet, as given by (4.4) and (4.8). 


\section{Intensity Ratios}

In this section we evaluate the ratio of the intensities of emitted second harmonic light and of the incident fundamental radiation. For each of the three geometries we consider the conversion coefficients (6.10). We assume that the fundamental is present as a single mode $k$ oscillating at frequency $\omega$ with amplitude characterized by $a_{1 k}^{-\mathrm{E}}(\omega)$ for TE-polarization and by $a_{1 k}^{-\mathrm{M}}(\omega)$ for TM-polarization. According to (4.1) for TEpolarization the electric field is given by

$\mathbf{E}(\omega ; x, z)=a_{1 k}^{-\mathrm{E}}(\omega) \psi_{k}^{\mathrm{E}}(\omega, z) \mathbf{e}_{y} \exp \left[i p_{k}^{\mathrm{E}}(\omega) x\right]$.

Assuming an isotropic tensor $\chi$ with mirror symmetry we find from (5.1) and (5.4) for the induced surface polarization

$$
\begin{aligned}
& \mathbf{P}^{\mathrm{SE}}(2 \omega, x) \\
& =\chi_{2} g_{N}(x)\left[a_{1 k}^{-\mathrm{E}}(\omega) \psi_{k}^{\mathrm{E}}\left(\omega, z_{0}\right)\right]^{2} \mathbf{e}_{z} \exp \left[2 \mathrm{i} p_{k}^{\mathrm{E}}(\omega) x\right]
\end{aligned}
$$

with $\chi_{2}=\chi_{z x x}=\chi_{z y y}$. For TM-polarization the electric field is according to (4.5)

$$
\begin{aligned}
\mathbf{E}(\omega ; x, z)= & \frac{a_{1 k}^{-\mathrm{M}}(\omega)}{k \varepsilon(\omega, z)}\left[-\mathrm{i} \frac{\partial \psi_{k}^{\mathrm{M}}(\omega, z)}{\partial z} \mathbf{e}_{x}\right. \\
& \left.-p_{k}^{\mathrm{M}} \psi_{k}^{\mathrm{M}}(\omega, z) \mathbf{e}_{z}\right] \exp \left[\mathrm{i} p_{k}^{\mathrm{M}}(\omega) x\right] .
\end{aligned}
$$

The induced surface polarization is

$$
\begin{aligned}
\mathbf{P}^{\mathrm{SM}}(2 \omega, x)= & g_{N}(x)\left[\frac{a_{1 k}^{-\mathrm{M}}(\omega)}{k \varepsilon(\omega, z)}\right]^{2}\left\{2 \mathrm{i} \chi_{3} p_{k}^{\mathrm{M}} \psi_{k}^{\mathrm{M}}(\omega, z) \frac{\partial \psi_{k}^{\mathrm{M}}(\omega, z)}{\partial z} \mathbf{e}_{x}\right. \\
& \left.+\left[\chi_{1}\left(p_{k}^{\mathrm{M}} \psi_{k}^{\mathrm{M}}(\omega, z)\right)^{2}-\chi_{2}\left(\frac{\partial \psi_{k}^{\mathrm{M}}(\omega, z)}{\partial z}\right)^{2}\right] \mathbf{e}_{z}\right\}\left.\right|_{z=z_{0}} \exp \left[2 \mathrm{i} p_{k}^{\mathrm{M}}(\omega) x\right]
\end{aligned}
$$

where $\chi_{1}=\chi_{z z z}$ and $\chi_{3}=\chi_{x z x}=\chi_{x x z}$. It follows from (4.4) and (4.8) that in both cases the emitted radiation is TM-polarized. For TE-polarized fundamental the emitted radiation is proportional to

$$
\begin{aligned}
& \left|\hat{P}_{z}^{\mathrm{SE}}\left(2 \omega, p_{j}^{\mathrm{M}}(2 \omega)\right)\right|^{2} \\
& =\left|\chi_{2}\right|^{2}\left|\hat{G}_{N}\left(p_{j}^{\mathrm{M}}(2 \omega)-2 p_{k}^{\mathrm{E}}(\omega)\right)\right|^{2}\left|a_{1 k}^{-\mathrm{E}}(\omega)\right|^{4}\left[\psi_{k}^{\mathrm{E}}(\omega, 0+)\right]^{4} .
\end{aligned}
$$

Here the amplitude factor may be expressed in terms of the intensity of the incident radiation by use of (3.6). This yields for the conversion coefficient $\eta_{j k}^{\mathrm{ME}}$ defined in (6.10)

$\eta_{j k}^{\mathrm{ME}}=256 \pi^{5} \omega^{-1}\left|\hat{G}_{N}\left(p_{j}^{\mathrm{M}}(2 \omega)-2 p_{k}^{\mathrm{E}}(\omega)\right)\right|^{2} A_{j k}\left|\chi_{2}\right|^{2} J_{k}^{\mathrm{E}}(\omega)$,

where the coefficient $A_{j k}$ is given by

$A_{j k}=C_{j k}^{\mathrm{ME}} a_{j k}$

with the normalization factor

$$
C_{j k}=p_{j}(2 \omega) p_{k}^{2}(\omega)\left[\varepsilon_{3}^{2}(2 \omega) \varepsilon_{3}^{4}(\omega) N_{j}(2 \omega) N_{k}^{2}(\omega)\right]^{-1}
$$

and the dimensionless wavefunction coefficient

$a_{j k}=\left[\varepsilon_{3}(\omega) k / p_{k}^{\mathrm{E}}(\omega)\right]^{4}\left[\psi_{j}^{\mathrm{M}}(2 \omega, 0+)\right]^{2}\left[\psi_{k}^{\mathrm{E}}(\omega, 0+)\right]^{4}$

Similarly we find for the conversion coefficient $\eta_{j k}^{\mathrm{MM}}$

$$
\begin{aligned}
\eta_{j k}^{\mathrm{MM}}= & 256 \pi^{5} \omega^{-1}\left|\hat{G}_{N}\left(p_{j}^{\mathrm{M}}(2 \omega)-2 p_{k}^{\mathrm{M}}(\omega)\right)\right|^{2}\left(\sum_{\lambda \mu} B_{j k}^{\lambda \mu} \chi_{\lambda} \chi_{\mu}^{*}\right) \\
& \times J_{k}^{\mathrm{M}}(\omega),
\end{aligned}
$$

where the coefficients $B_{j k}^{\lambda \mu}$ are given by

$B_{j k}^{\lambda \mu}=C_{j k}^{\mathrm{MM}} b_{j k}^{\lambda \mu}$

with normalization factor $C_{j k}^{\mathrm{MM}}$ given by (8.8) and dimensionless wavefunction coefficient $b_{j k}^{\lambda \mu}$ given by

$b_{j k}^{11}=\psi_{j}^{2} \psi_{k}^{4}, \quad b_{j k}^{12}=p_{k}^{-2} \psi_{j}^{2} \psi_{k}^{2} \psi_{k}^{\prime 2}$,

$b_{j k}^{13}=2 p_{j}^{-1} p_{k}^{-1} \psi_{j} \psi_{j}^{\prime} \psi_{k}^{3} \psi_{k}^{\prime}$,

$b_{j k}^{22}=p_{k}^{-4} \psi_{j}^{2} \psi_{k}^{\prime 4}$,

$b_{j k}^{23}=-2 p_{j}^{-1} p_{k}^{-3} \psi_{j} \psi_{j}^{\prime} \psi_{k} \psi_{k}^{\prime 3}$,

$b_{j k}^{33}=4 p_{j}^{-2} p_{k}^{-2} \psi_{j}^{\prime 2} \psi_{k}^{2} \psi_{k}^{\prime 2}$.

The values of the wavefunction and its derivative at $z_{0}=0+$ are given by (7.12) and (7.13).

We are now in a position to compare the conversion coefficients for different geometries. We assume that for each of the geometries only the lowest fundamental mode is excited with the same intensity
$J_{0}^{\mathrm{E}}(\omega)$ or $J_{0}^{\mathrm{M}}(\omega)$ and we consider the intensity $J_{0}^{\mathrm{M}}(2 \omega)$ of the lowest second harmonic mode. Assuming perfect phase-matching the factor $F_{N}$ in (5.9) takes the value $N^{2}$ and choosing $b=\frac{1}{2} a$ the first factor in (5.7) has absolute value $a \sqrt{2} / 4 \pi^{2}$. In this case the three geometries differ only in the factors $A_{00}$ and $B_{00}^{\lambda \mu}$ in (8.6) and (8.10).

In our numerical calculations we put the magnetic permeability $\mu$ equal to unity everywhere. At the fundamental frequency we put $\varepsilon_{1}(\omega)=2.13, \varepsilon_{2}(\omega)$ $=2.25$, and $\varepsilon_{3}(\omega)=1$. At the second harmonic frequency we put $\varepsilon_{1}(2 \omega)=2.17, \varepsilon_{2}(2 \omega)=2.28$, and $\varepsilon_{3}(2 \omega)=1$. In Fig. 5 we plot the reduced wavenumber $p_{0}(\omega) / k$ for the three geometries as a function of $k d$. The corresponding plot for $p_{0}(2 \omega) / 2 k$ at the second harmonic frequency is very similar. In Fig. 6 we plot the logarithm of the ratio $\left(A_{00} / 4 k^{6}\right)$ as a function of $k d$. In Fig. 7 we plot the logarithm of the ratio $\left(B_{00}^{\mathbf{1 1}} / 4 k^{6}\right)$, again as a function of $k d$. We note that in geometry III the derivatives $\psi_{0}^{\prime}(\omega, 0+)$ and $\psi_{0}^{\prime}(2 \omega, 0+)$ vanish by symmetry, so that 


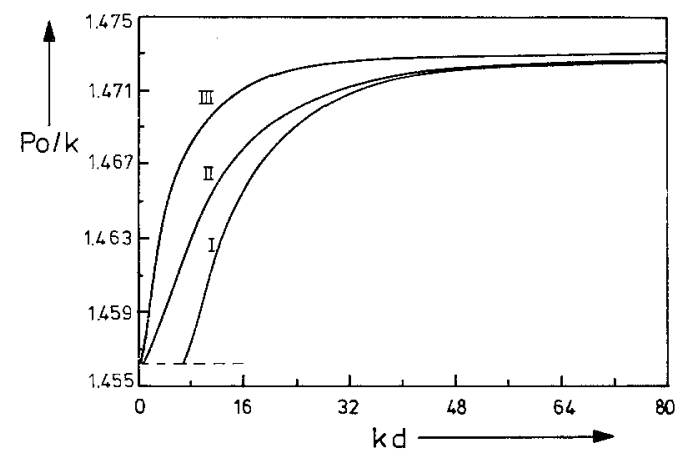

Fig. 5. Reduced wavenumber $p_{0}(\omega) / k$ for the fundamental guided mode of lowest order plotted against $k d$ for geometries I, II, and III

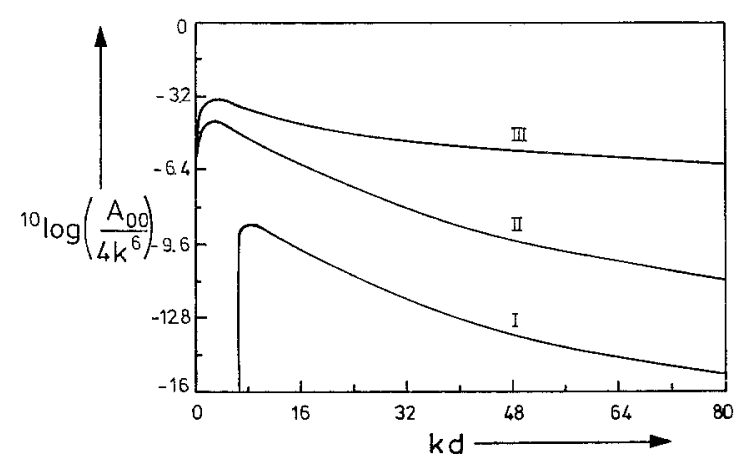

Fig. 6. The logarithm ${ }^{10} \log \left(A_{00} / 4 k^{6}\right)$, with $A_{00}$ as defined in (8.7), for geometries I, II, and III plotted against $k d$

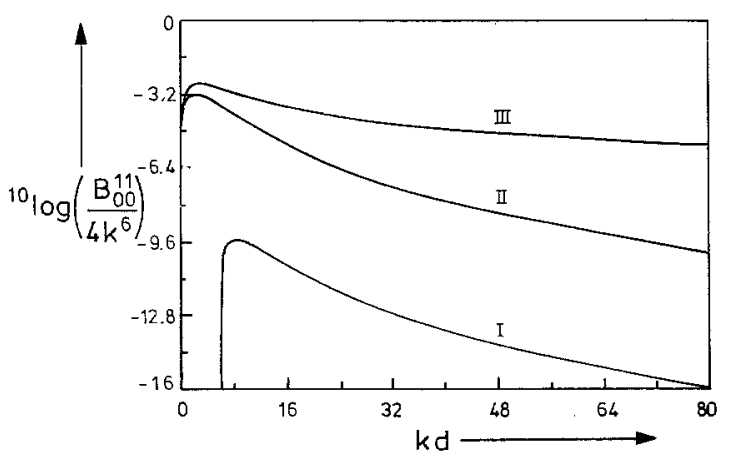

Fig. 7. The logarithm ${ }^{10} \log \left(B_{00}^{11} / 4 k^{6}\right)$, with $B_{00}^{11}$ as defined in (8.11), for geometries I, II, and III plotted against $k d$.

in (8.12) only the coefficient $b_{00}^{11}$ differs from zero. It is evident from Figs. 6 and 7 that there is a marked difference in efficiency for the various geometries. Roughly speaking geometry II is several orders of magnitude more efficient than geometry I, and geometry III is again several orders of magnitude more efficient than geometry II.

In all three geometries the distribution of the adsorbate enters via the prefactor $\left|\widehat{G}_{N}\left(p_{j}(\omega)-2 p_{k}(\omega)\right)\right|^{2}$ in (8.6) and (8.10). A less than perfect grating affects this geometrical prefactor. Also irregularities in the waveguide structure affect mainly this prefactor. If we express the effect of scattering due to irregularities by an exponential damping of the wave, i.e. by complex values of the guided mode wavenumbers $p_{j}(2 \omega)$ and $p_{k}(\omega)$, then both the surface polarization and the second harmonic wave are exponentially damped in the $x$-direction. In calculating the second harmonic intensity at a distance $D$ from the grating we must replace the factor $\left|\hat{G}_{N}\right|^{2}$ by

$\exp \left[-2 p_{j}^{\prime \prime}(2 \omega)(D+N a)\right]\left|\widehat{G}_{N}\left(p_{j}(2 \omega)-2 p_{k}(\omega)\right)\right|^{2}$,

where now $p_{j}=p_{j}^{\prime}+\mathbf{i} p_{j}^{\prime \prime}$ and $p_{k}=p_{k}^{\prime}+\mathbf{i} p_{k}^{\prime \prime}$ are complex wavenumbers. In order to find the optimum grating we may maximize the above expression with respect to $N$.

\section{Efficiency Considerations}

In this section we compare the conversion efficiency of the various waveguide configurations studied in the preceding sections with that of bulk-SHG in KDP. First we compare surface-SHG and bulk-SHG for the case of Fresnel reflection (FR) and total internal reflection (TIR) at a single surface. Next we consider the conversion efficiency for the different waveguide configurations for realistic physical dimensions and under the assumption that recently available nonlinear monolayers of high second-order susceptibility $\chi_{\mathrm{s}}^{(2)}$ are employed.

In order to facilitate the comparison of surfaceSHG and bulk-SHG we assume that in the former case the incident wave is s-polarized. Hence the SHG signal is p-polarized and depends only on the surface susceptibility component [28] $\chi_{z y y}$. The surface susceptibility $\chi_{\mathrm{s}}^{(2)}$ can be as high as $10^{-13}$ esu [29]. Typically the bulk susceptibility $\chi^{(2)}$ of KDP approaches $10^{-8}$ esu [30]. For a particular choice of geometry in surface-SHG we may evaluate the effective length $l_{\text {eff }}$ of a KDP crystal leading to the same conversion efficiency under the assumption of perfect phase-matching [31]. Table 1 represents a compilation of such effective KDP-lengths for different angles of incidence in the FR or TIR case. It is evident from this table that surface-SHG can be quite an efficient conversion process. In fact, for an angle of incidence of $45^{\circ}$ in Fresnel reflection and for $\chi_{\mathrm{s}}^{(2)}=10^{-13} \mathrm{esu}$ it takes $790 \mathrm{~nm}$ phase-matched KDP to achieve the same conversion efficiency. The enhancement by an additional factor 10 in total reflection is in agreement with previous measurements $[32,33]$. This enhancement is reduced considerably upon deviation from the critical angle of incidence. Under the conditions of Table 1 this angle is $43.3^{\circ}$ for $\varepsilon(\omega)=2.13$. However, even for $60^{\circ}$ incidence the length $l_{\text {eff }}$ in total reflection still exceeds the value for Fresnel reflection. 
Table 1. Effective KDP-lengths in nanometer for Fresnel reflection (FR) and total internal reflection (TIR) for selected angles of incidence of a surface-SHG experiment (see text for details)

\begin{tabular}{lll}
\hline $\begin{array}{l}\text { Angle of } \\
\text { Incidence }\end{array}$ & $l_{\text {eff }}$ for KDP & $l_{\text {eff }}$ for KDP \\
& FR $[\mathrm{nm}]$ & TIR [nm] \\
\hline $45^{\circ}$ & 797 & 7906 \\
$46^{\circ}$ & 807 & 7411 \\
$47^{\circ}$ & 817 & 6988 \\
$48^{\circ}$ & 826 & 6551 \\
$49^{\circ}$ & 824 & 6170 \\
$50^{\circ}$ & 842 & 5816 \\
$60^{\circ}$ & 847 & 3149 \\
\hline
\end{tabular}

In Fig. 8 we plot the calculated conversion efficiencies for the three previously discussed waveguide configurations I, II, and III as a function of the waveguide length $N$. a. An initial cross section $A=0.5 \mu \mathrm{m} \times 0.5 \mu \mathrm{m}=0.25 \mu \mathrm{m}^{2}$ and a typical power of $100 \mathrm{~mW}$ in the red spectral range have been assumed. We compare with the conversion efficiency of KDP as calculated under optimized focusing conditions for Gaussian beams [30], which results in a linear increase with crystal length. In the waveguide devices the conversion efficiency will exceed the linear dependence with length due to continuous guidance in the $z$-direction of the fundamental wave. However, the quadratic dependence shown in Fig. 8 will not always be realized, since the fundamental wave will tend to spread out in the $y$-direction. In Fig. 8 we have assumed a lateral spreading of the fundamental beam identical to that in KDP for the same length. According to Fig. 8 a conversion efficiency of $0.01 \%$ at an input power of $100 \mathrm{~mW}$ seems achievable for waveguide version III. Provided a critical length is exceeded the efficiency of the device is higher than a KDP crystal of the same length. It should be added that the proposed device is open to improvements. We have assumed a $\chi_{\mathrm{s}}^{(2)}$-value of $10^{-13}$ esu. Newly synthesized organic dyes [34] may exhibit surface susceptibilities as high as $5 \times 10^{-13}$ esu, or even $10^{-12}$ esu, under favorable excitation conditions. These large nonlinearities should compensate for the inevitable losses due to absorption of the generated SH-radiation and due to scattering of the fundamental. The experimental results of $[24,30]$ clearly indicate that absorption-free guidance of the fundamental wave in adsorbatecovered waveguide structures is possible. In addition, latest developments in waveguide fabrication [35] indicate that guidance of the fundamental in both the vertical and lateral directions is now possible. The high nonlinearity achievable with surface effects, continuous large-scale phase-matching, and possibly two-

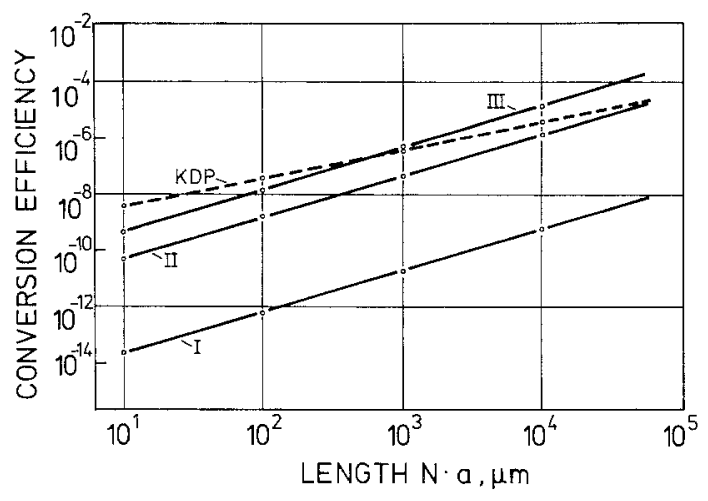

Fig. 8. Conversion efficiency versus length for the various waveguide configurations and KDP. For each of the three waveguide geometries we choose a thickness $d$ corresponding to the maximum in Fig. 6 and we assume perfect phase-matching. For KDP we assume optimized focussing conditions according to $[25]$

dimensional confinement of the fundamental wave should ultimately allow higher conversion efficiencies as compared to conversion with inorganic crystals.

\section{Discussion}

We have discussed in some detail the relative merits of three different geometries for second harmonic generation in planar optical waveguides. In all three geometries, shown in Figs. 1, 3, and 4, we consider a monolayer of optically nonlinear molecules adsorbed at the interface between media 2 and 3 arranged in a periodic grating structure. We have found that the symmetric geometry shown in Fig. 4 is by far the most efficient. Experimental efforts should aim at realizing this favourable geometry.

We have simplified our calculation by the assumption that in the excitation of the nonlinear surface polarization by the fundamental wave depletion of the latter may be neglected. If the proposed mechanism is efficient and an appreciable part of the fundamental intensity is converted into second harmonic intensity, then the above assumption can no longer be justified and a more complicated coupled wave theory must be considered [17-19].

\section{References}

1. G.I. Stegeman, C.T. Seaton: J. Appl. Phys. 58, R57 (1985) G.I. Stegeman, R.H. Stolen: J. Opt. Soc. Am. B 6, 652 (1989)

2. J.E. Sipe, G.I. Stegeman, C. Karaguleff, R. Fortenberry, R. Moshrefzadeh, W.M. Hetherington III, N.E. Van Wyck: Opt. Lett. 8, 461 (1983)

3. J.E. Sipe, G.I. Stegeman: J. Opt. Soc. Am. 69, 1676 (1979) 
4. E.M. Conwell: IEE J. QE-9, 867 (1973)

5. K. Jain, G.H. Hewig: Opt. Commun. 36, 483 (1981)

6. D.J. Williams: In Nonlinear Optical Properties of Organic Molecules and Crystals, Vol. 1, ed. by D.S. Chemla, J. Zyss (Academic, Orlando 1987) p. 405

7. B.F. Levine, C.G. Bethea, R.A. Logan: Appl. Phys. Lett. 26, $375(1975)$

8. B.U. Chen, C.C. Ghizoni, C.L. Tang: Appl. Phys. Lett. 28, 651 (1976)

9. H.A. Haus, G.A. Reider: Appl. Opt. 26, 4576 (1987)

10. G.A. Reider, M. Huemer, A.J. Schmidt: Opt. Commun. 68, 149 (1988)

11. R. Regener, W. Sohler: J. Opt. Soc. Am. B 5, 267 (1988)

12. T. Taniuchi, K. Yamamoto: Optoelectron Devices and Technologies 2, 53 (1987)

13. B. Jaskorzynska, G. Arvidsson, F. Laurell: Proc. SPIE 651, 221 (1986)

14. E.J. Lim, M.M. Fejer, R.L. Byer: Electron. Lett. 25, 174(1989)

15. J. Webjörn, F. Laurell, G. Arvidsson: In Topical Meeting on Nonlinear Guided-Wave Phenomena: Physics and Applications, 1989 Technical Digest Series, Vol. 2 (Optical Society of America, Washington D.C. 1989) p. 6

16. M. Born, E. Wolf: Principles of Optics (Pergamon, Oxford 1975) p. 51

17. D. Marcuse: Theory of Dielectric Optical Waveguides (Academic, New York 1974)

18. H. Kogelnik: In Integrated Optics, Topics Appl. Phys. 7, ed. by T. Tamir (Springer, Berlin, Heidelberg 1979) p. 13
19. H.A. Haus: Waves and Fields in Optoelectronics (Prentice Hall, Englewood Cliffs, NJ 1984)

20. L.D. Landau, E.M. Lifshitz: Quantum Mechanics (Pergamon, Oxford 1965) p. 492

21. B.U. Felderhof, G. Marowsky: Appl. Phys. B 43, 161 (1971)

22. V. Mizrahi, J.E. Sipe: J. Opt. Soc. Am. B 5, 660 (1988)

23. E.L. Ince: Ordinary Differential Equations (Dover, New York 1956) p. 123

24. B.U. Felderhof, G. Marowsky: Appl. Phys. B 44, 11 (1987)

25. J.M. Chen, J.R. Bower, C.S. Wang, C.H. Lee: Opt. Commun. 9, 132 (1973)

26. C.K. Chen, T.F. Heinz, D. Ricard, Y.R. Shen: Phys. Rev. Lett. 46, 1010 (1981)

27. B. Dick: Chem. Phys. 96, 199 (1985)

28. G. Marowsky, A. Gierulski, R. Steinhoff, D. Dorsch, R. Eidenschink, B. Rieger: J. Opt. Soc. Am. B 4, 956 (1987)

29. G. Marowsky, L.F. Chi, D. Möbius, R. Steinhoff, Y.R. Shen, D. Dorsch, B. Rieger: Chem. Phys. Lett. 147, 420 (1988)

30. Y.R. Shen: The Principles of Nonlinear Optics (Wiley, New York 1986) p. 88

31. For definition of all factors entering the expressions for SHG via surface effects see [24] and [Ref. 30, p. 496]

32. G. Marowsky, A. Gierulski, B. Dick: Opt. Commun. 52, 339 (1985)

33. A. Gierulski: Dissertation, Universität Hannover (1986)

34. G. Marowsky, G. Lüpke, R. Steinhoff: Submitted to Phys. Rev. Lett.

35. R. Ross: Private communication 